\title{
Research Paper: The Survey and Comparison of Musculoskeletal Disorders of Shoulder, Arm and Hand in Taxi and Bus Drivers in the City of Shiraz in 2016
}

\author{
Shohreh Taghizadeh $\oplus^{1}$, Farzaneh Haghighat ${ }^{1}$, ${ }^{*}$ Soraya Piroozi ${ }^{1}$, Azad Karimi ${ }^{1}$, Danial Khanali Nejad ${ }^{1}$
}

1. Department of Physiotherapy, School of Rehabilitation Sciences, Shiraz University of Medical Sciences, Shiraz, Iran.

\begin{tabular}{|c|c|}
\hline $\begin{array}{l}\text { Use your device to scan } \\
\text { and read the article online }\end{array}$ & CFtation: Taghizadeh Sh, Haghighat F, Piroozi S, Karimi A, Khanali Nejad D. [The Survey and Comparison of Musculoskeletal \\
\hline 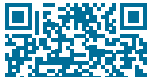 & $\begin{array}{l}\text { Disorders of Shoulder, Arm and Hand in Taxi and Bus Drivers in the City of Shiraz in } 2016 \text { (Persian)]. Archives of Rehabilitation. } \\
\text { 2018; 19(1):64-75. https://doi.org/10.21859/JREHAB.19.1.64 }\end{array}$ \\
\hline 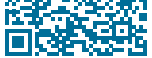 & dol': https://doi.org/10.21859/JREHAB.19.1.64 \\
\hline
\end{tabular}

Received: 03 Aug. 2017 Accepted: 20 dec. 2017

Keywords: WMSDs, DASH, Questionnaire, Bus driver, Taxi driver

\begin{abstract}
Objective Musculoskeletal disorders are a wide range of symptoms including pain and disability that are related to workspace and devices. Repeated activity, adopting fixed and inappropriate postures for a long time and activities including powerful movements of extremities are factors associated with WRMSDs. It seems professional drivers suffer from shoulder and upper extremities symptoms because of repetitive and long working hours with steering wheel and gear. The aim of this study was to investigate and compare the shoulder, arm, and hand disability among bus and taxi drivers.

Materials \& Methods This research was a cross-sectional, descriptive analytic study. In this study, the prevalence of upper limb musculoskeletal disorders in the urban bus and taxi drivers was investigated and compared. Subjects were included 300 professional drivers aged $25-55$ years (mean $\pm S D=41.24 \pm 8.28$ for bus drivers and $41.22 \pm 8.09$ for taxi drivers) who were divided into two groups ( 150 bus drivers and 150 taxi drivers). The method of sampling was simple. The inclusion criteria were: two years' experience in professional driving and driving for eight hours or two four-hours a day for four days a week. Shoulder, arm, and hand disability were assessed based on DASH and a demographic questionnaire. DASH questionnaire asks about individual's symptoms (such as pain severity during sleep or activity, weakness, and stiffness) as well as their ability to perform certain activities (individuals' problem in daily activities and also social and occupational activities). Statistical analysis was performed by SPSS 21, t-test, Chi-square, and Spearman correlation tests.

Results According to statistical findings, $33.66 \%$ of taxi drivers and $33.67 \%$ of bus drivers had musculoskeletal disorders of the shoulder, arm, and hand. In bus drivers, $46.66 \%, 48.66 \%$ and $4.66 \%$ and in taxi drivers $47.33 \%$, $45.33 \%, 6.66 \%$ had no, light, and moderate problem in daily activities, respectively and just $0.66 \%$ of taxi drivers had severe problems in these activities. In bus drivers, $32.66 \%, 64 \%$ and $3.33 \%$ and in taxi drivers $34.66 \%$, $60.66 \%$ and $4.66 \%$ had no, light, and moderate symptoms, respectively. In bus drivers, $36 \%, 40.66 \%, 18 \%$, $5.33 \%$ and in taxi drivers $36 \%, 41.33 \%, 18 \%, 4 \%$ had no, light, moderate and severe problems in occupational and social activities, respectively and just $0.66 \%$ of taxi drivers were unable to perform these activities. There was a correlation between work hours per week $(r=0.24, P=0.003)$ hours of sleep per day and night $(r=0.24$, $P=0.003)$ and age $(r=0.16, P=0.041)$ with musculoskeletal disorders in bus drivers. Also, there was a correlation between hours of sleep per day and night and musculoskeletal disorders in taxi drivers $(r=0.19, P=0.016)$. There was no significant difference between disability, symptoms, and function of taxi and bus drivers ( $P=0.97)$ Conclusion This study demonstrated increasing the sleep hours and decreasing the work hours per day and week can reduce the possibility of shoulder, arm, and hand symptoms.
\end{abstract}




\title{
بررسى و مقايسه اختلالات اسكلتىعضلانى شانه، بازو و دست در رانندكّان تاكسى و اتوبوس

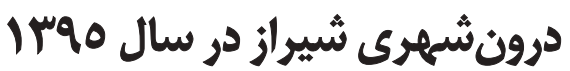

\author{
شهره تقىزاده'ه، فرزانه حقيقت'، "ثريا بيروزى'، آزاد كريمى'، دانيال خانعلىنزاد' \\ ا - كروه فيزيوترائي، دانشكده علوم توانبخشى، دانشكاه علوم يزٔشكى شيراز، شيراز، ايران.
}

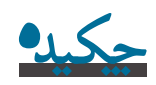

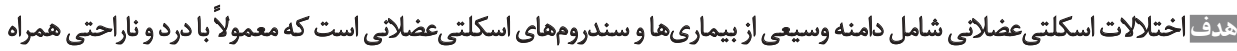

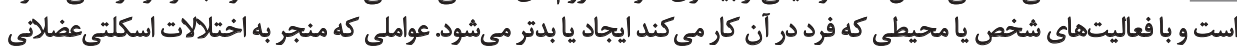

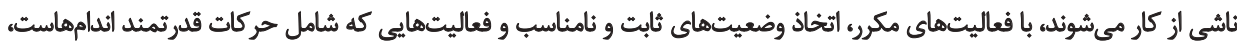

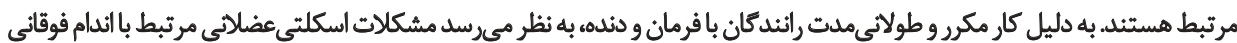

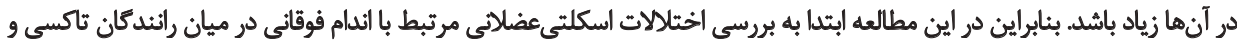

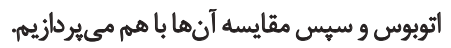

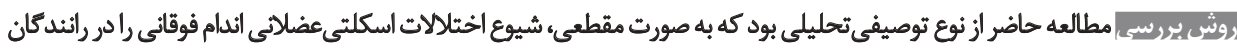

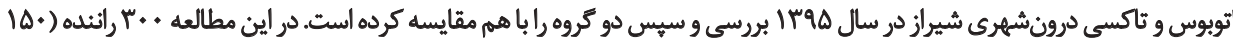

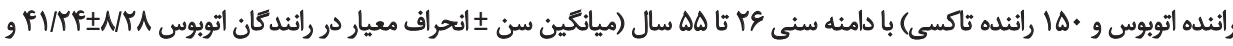

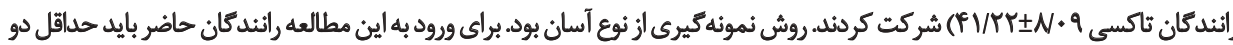

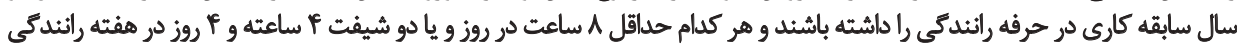

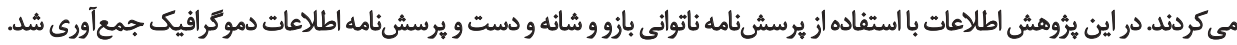

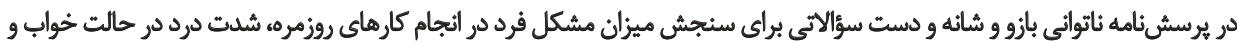

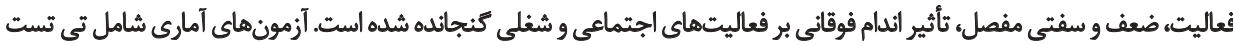

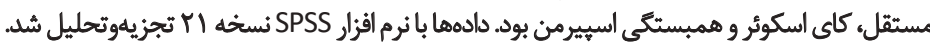

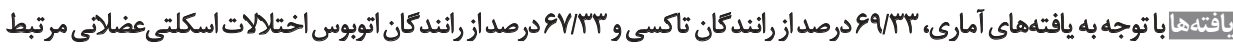

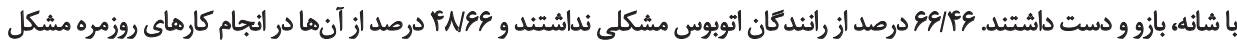

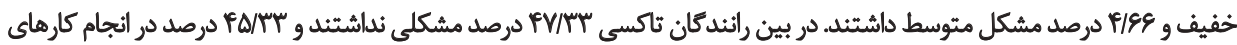

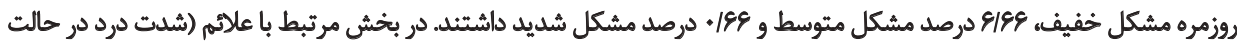

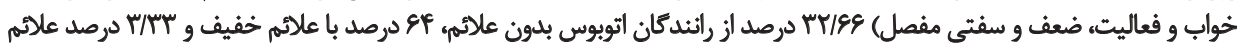

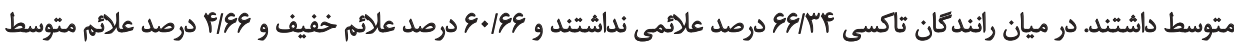

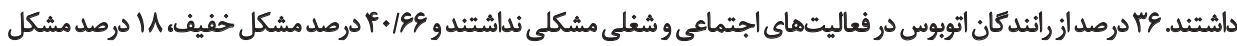

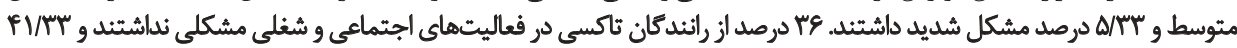

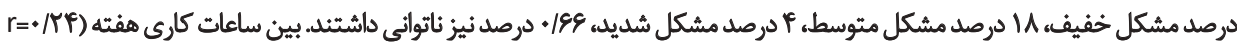

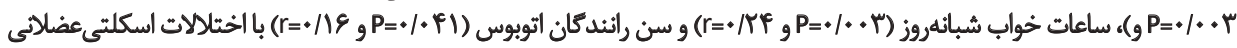

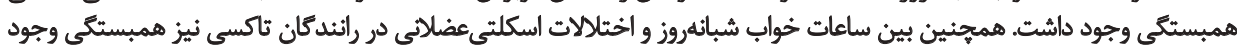

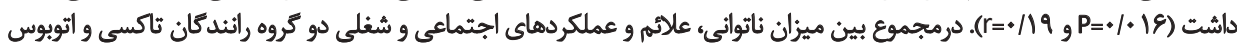

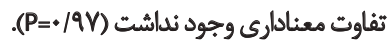

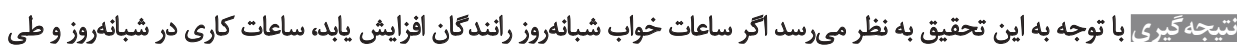

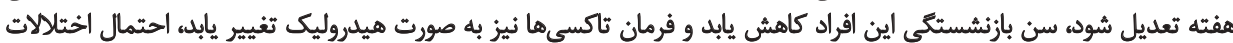

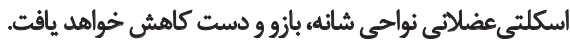

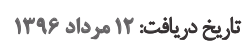

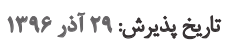

كليدواثوها:

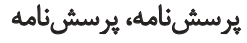

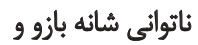
دست، اختلالات باتئ أسكلتى عضلانى مرتبط

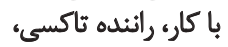
رانتده اتوبوس
\end{abstract}


طراحى نامناسب وسايل نثليه ممكن است منجر به مشكلاتى مقدمه

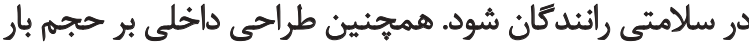

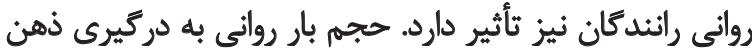

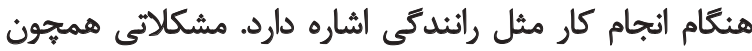

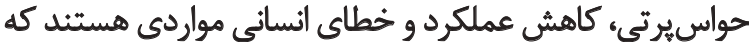
با حجم بار روانى مرتبط هستئد [r.

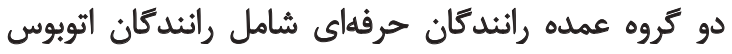

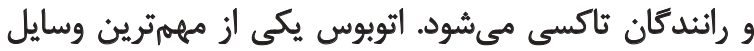

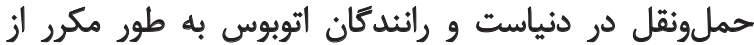

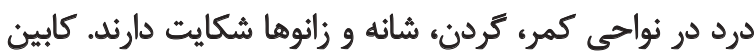

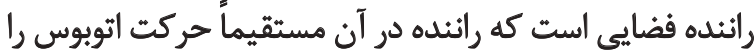

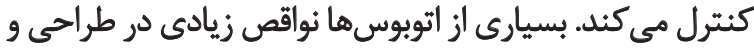

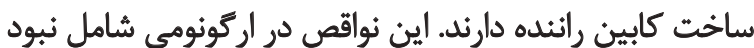

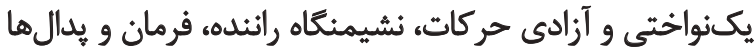

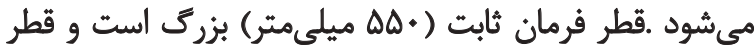

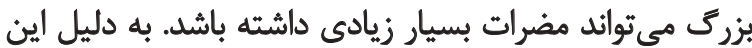

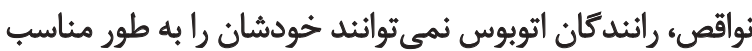

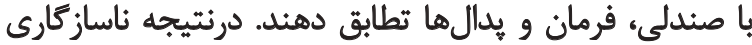

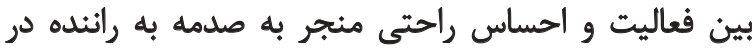

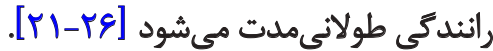

از طرفى تاكسىرانى نيز به عنوان يكى از مشاغل مهرم در

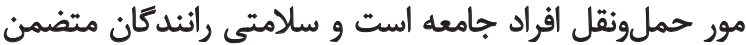

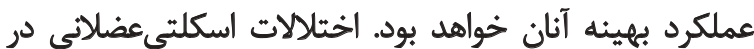

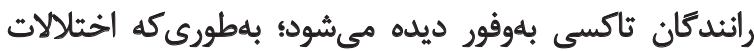

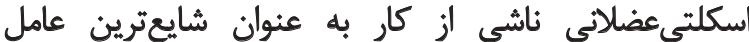

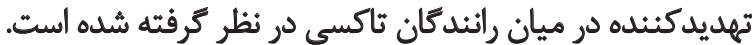

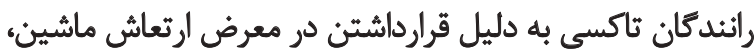

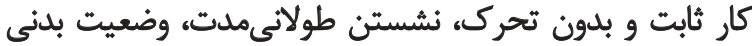

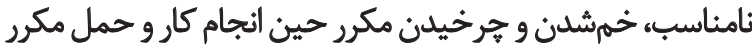

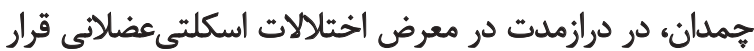

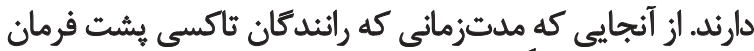

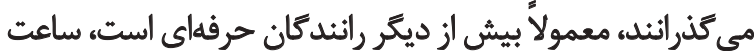

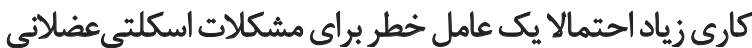

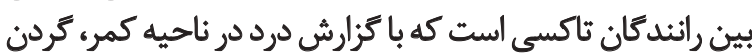

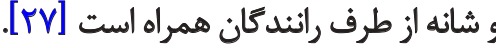

بررسى دردهاي اسكلتى عضلانى بين رانددكان تاكسى و اتوبوس

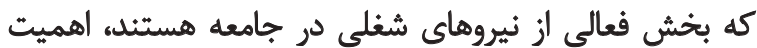

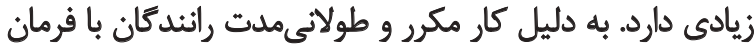

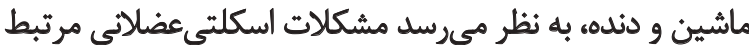

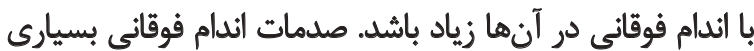

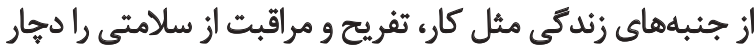

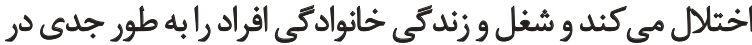

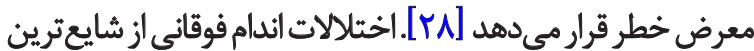

اختلالات اسكلتىعضلاتى' به شرايطى اطلاق ميشود كه

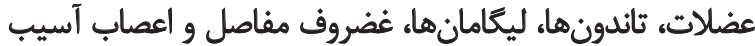

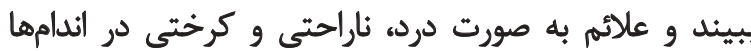

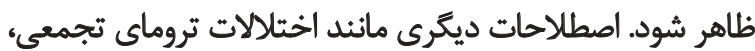

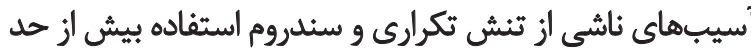
براى بيان اين شرايط استفاده مى تشود.

اختلالات اسكلتىعضلاتى مرتبط با كار ب اختلالاتى هستند

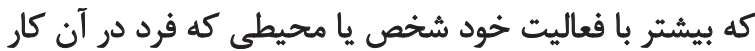

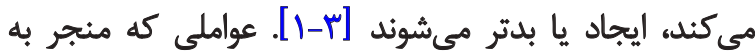

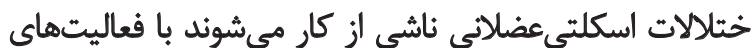

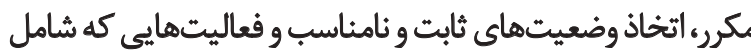

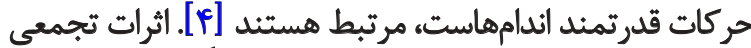

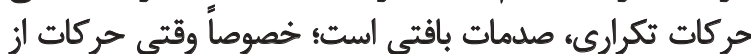

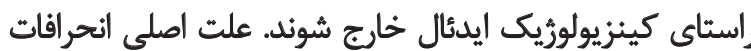
در الكوى حركتى مفصل، حركات تكرارى و حفظ وضئ وضعيتها

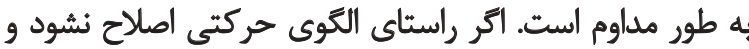

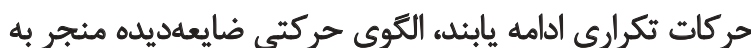

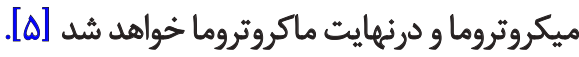
اختلالات اسكلتى عضلانى مرتبط با كار بيشتر در كمر، تردن،

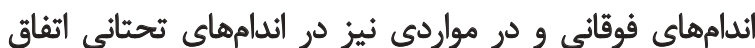

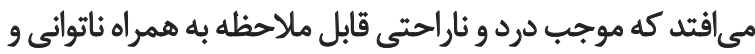

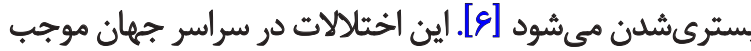

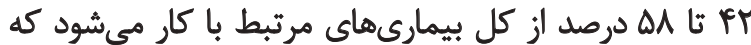

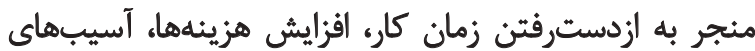

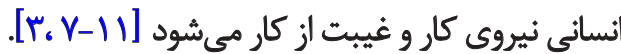

يكى از مشاغلى كه بيمارىهاى مرتبط با كار در آن ديده

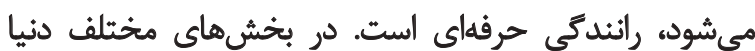

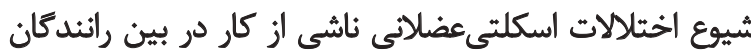

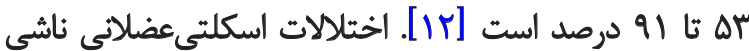

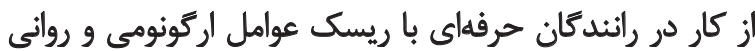

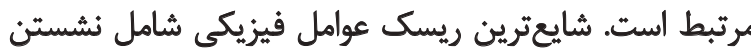

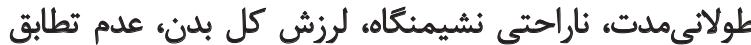

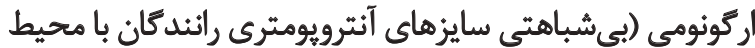

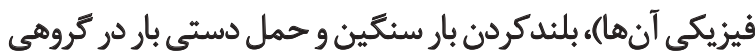

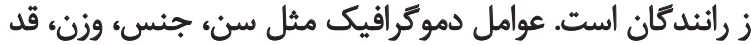

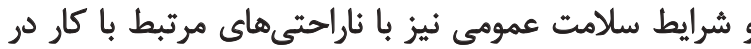

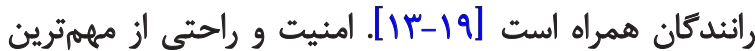

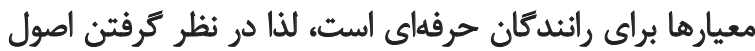

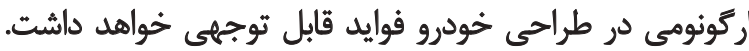

1. Musculo-Skeletal Disorders (MSDs)

2. Work-related Musculo-Skeletal Disorders (WMSDs) 


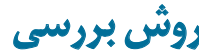

مطالعه حاضر از نوع توصيفىتحليلى است كه به صورت

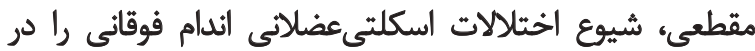

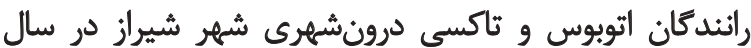

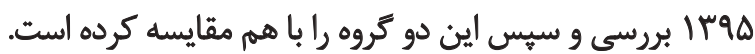

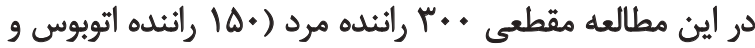

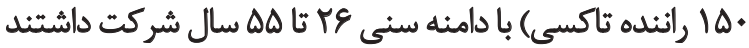

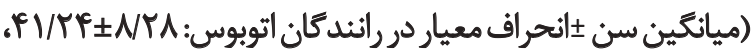

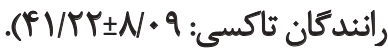

روش نمونهيرى از نوع آسان بود. براي ورود به اين مطالعه

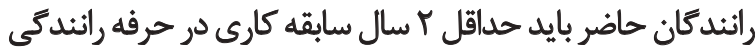

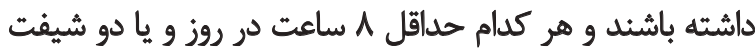

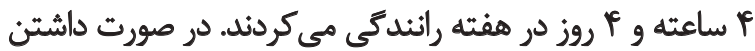

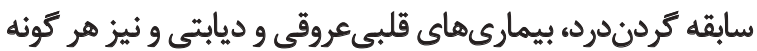

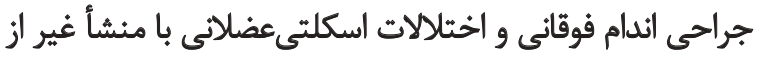

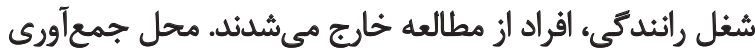

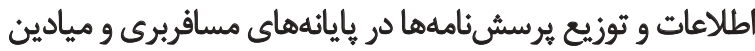

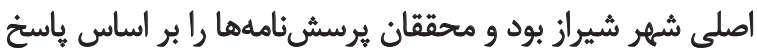

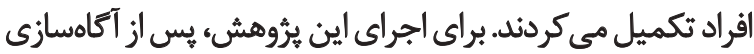

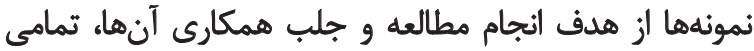
شركت كنيدكان فرم رضايتنامه اخلاقى را المضا كردئد.

اطلاعات مورد نياز از طريق مصاحبه با استفاده از دو يرسشنامه

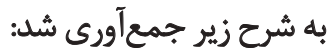

1. يرسشنامه اطلاعات دموكرافيك و شغلى رانندكان شامل

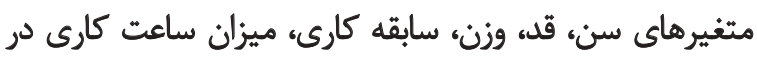

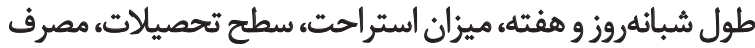

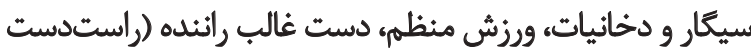
يا جيدست)، نوع فرمان (هيدروليك يا مكانيك) بود.

r. برسشنامه ناتوانى بازو و شانه و دست كه ميزان شيوع

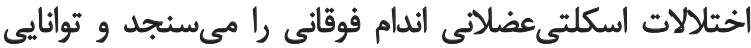

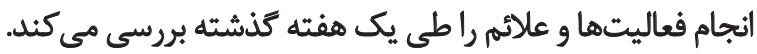

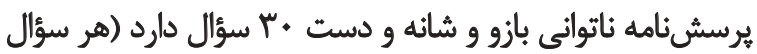

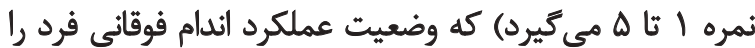

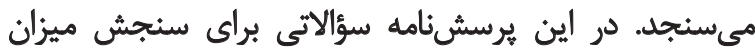

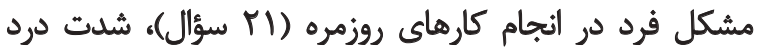

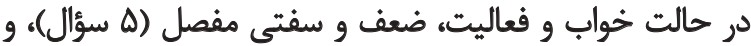

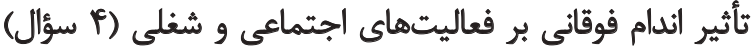

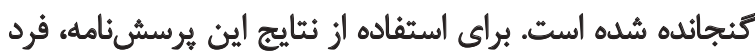

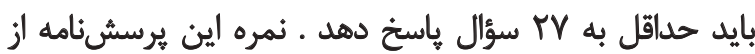

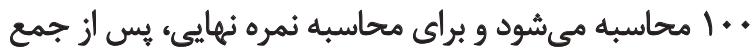

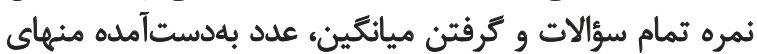

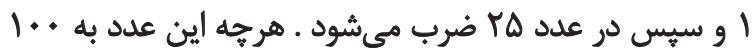

مشكلات عصبى عضلاتى اسكلتى است. با بهدست آوردن اطلاعات

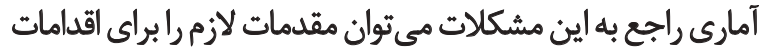

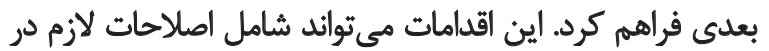

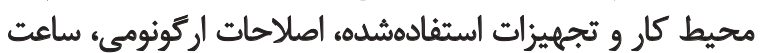

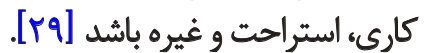

مطالعات صورت كرفته در ايران بيشتر عواملى رابررسى كردهاند

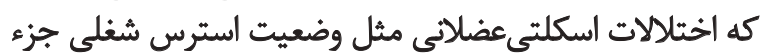

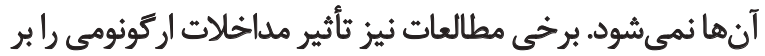

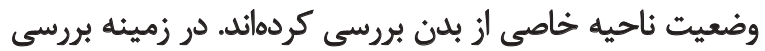

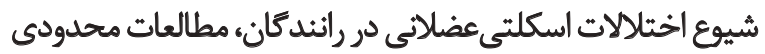

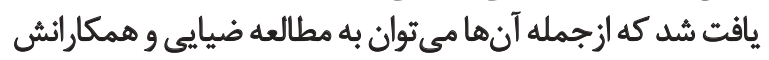

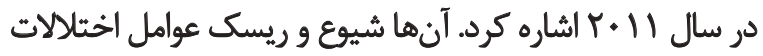

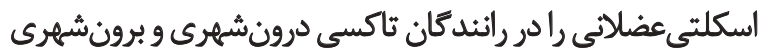

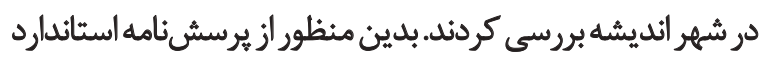

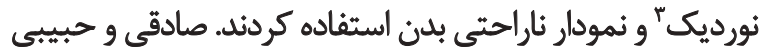

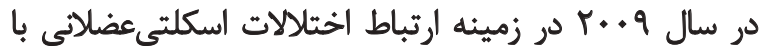

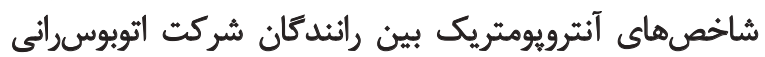

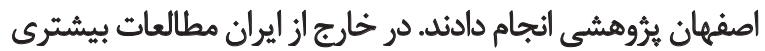

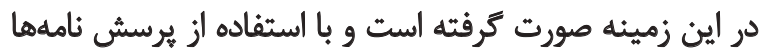

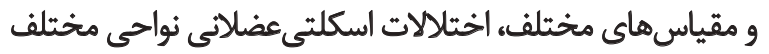

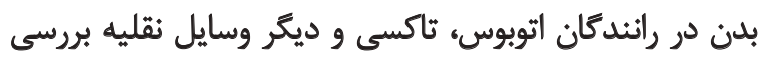

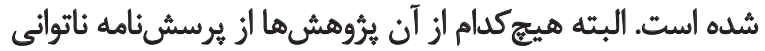

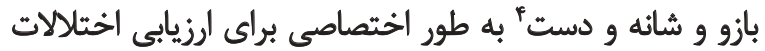

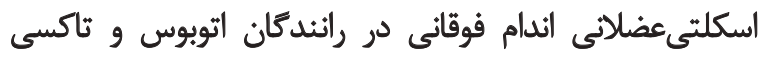

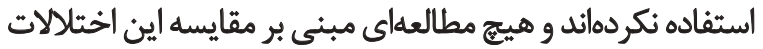
در اين دو كروه رانندكان يافت نشئد مطادئ

در اين مطالعه اختلالات اسكلتى عضلانى مرتبط با شانه، بازو و

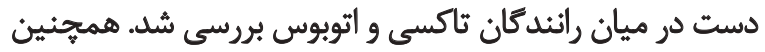

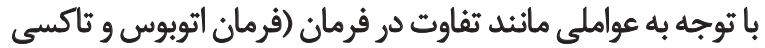

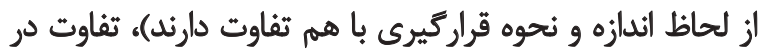

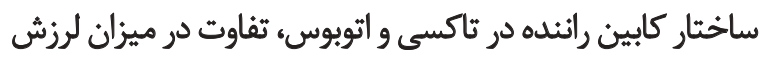

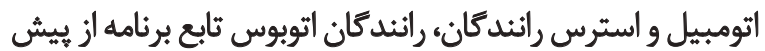

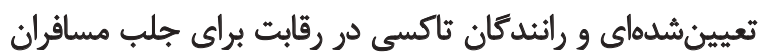

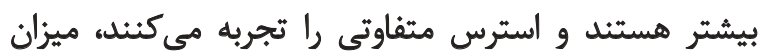

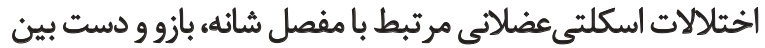

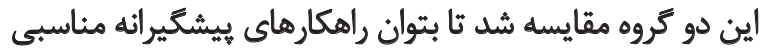
را به منظور كاهش اين اختلالات در جمعيت رانداندان رامكان ارائه دادئ

مديريث ايمن و مؤثر مستلزم توجه به عوامل انسانى و اجزاي

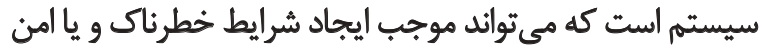

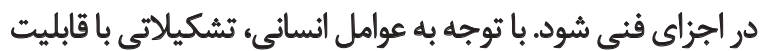

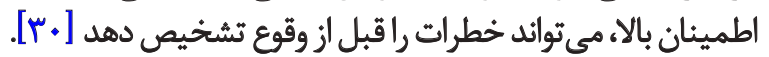

4. Disabilities of the Arm, Shoulder and Hand (DASH) 


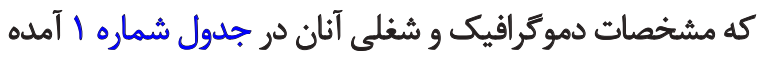

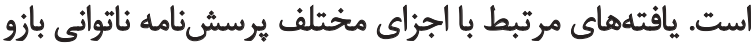

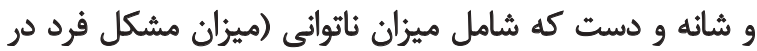

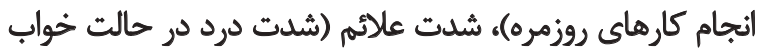

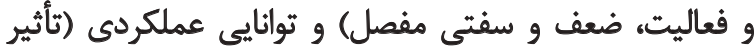

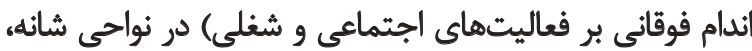

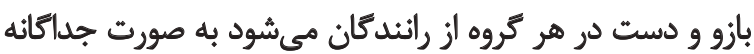

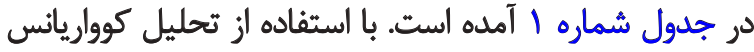

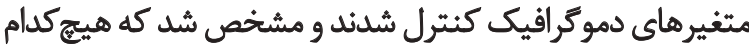

$$
\text { از متغيرهاي مذكور معنادار نبودند. }
$$

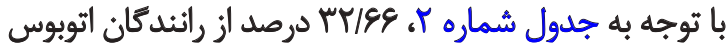

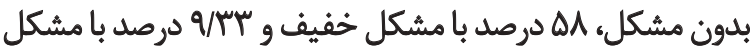

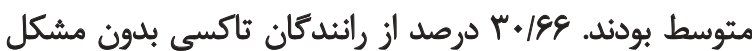

نزديكتر باشد، نشاندهنده ناتوانى بيشتر فرد است. موسوى و

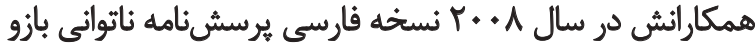

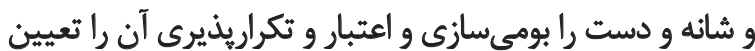

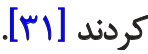

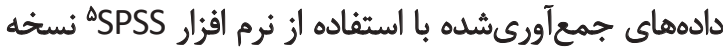

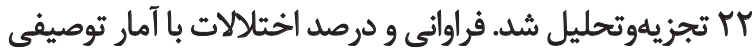

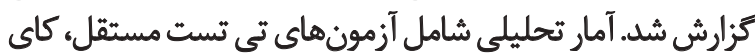

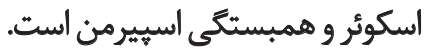

بافتلها

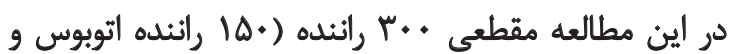

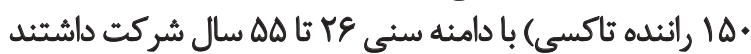

5. IBM SPSS Statistics

جدول ا. اطلاعات دموكرافيك و شغلى دو كروه رائدكان اتوبوس و ثاكسى (ميانكين \pm انحراف معيار)

\begin{tabular}{|c|c|c|c|c|c|c|c|}
\hline هيزان كار در طول & شيزانهروز (ساعت طول) & شبانهروز (سابعت در) & سابقه كار (سال) & وزن (كيلوكرم) & قد (متر) & سن (سال) & راندأنان \\
\hline$V / M E \pm r r / r q$ & $\mid r / \& \Delta \pm N \cdot r$ & $8 / \cdot \pm$ V/ar & $\mid F / g r \pm N A H$ & $W / 1 r \pm 11 / 4 q$ & $1 / M+ \pm+/ 8 \Lambda$ & $P I / T Y \pm N K A$ & أتوبوس \\
\hline$n / 11 \pm 10 / 8 q$ & $1 \cdot / Q_{0} \pm T / r \Delta$ & SNODI/AI & $\mid r / T \Delta \pm V / \Lambda \Delta$ & $A V / \cdot \pm \pm 1 D / A$ & $1 / M^{\prime \prime \pm+/ A r ~}$ & $P I / T \pm \pm N \cdot q$ & تاكسى \\
\hline$+/ Q A$ &.$/ 61^{\circ}$ & $+1++^{*}$ &.$/ 61 t^{2 *}$ & $+1+10^{*}$ &.$/ 94$ &.$/ 4$ & $P$ \\
\hline
\end{tabular}

توانبخنتى

جدول r. فراواني و درصد اختلالات اسكلتي عضلاتي نواحي شانه، بازو و دست در رائدكان اتوبوس و ثاكسي (نمره مجموع يرسشئامه ناتواني بازو و شانه و دست)

\begin{tabular}{|c|c|c|c|c|c|c|c|c|c|c|}
\hline \multicolumn{2}{|c|}{ 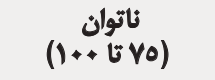 } & \multicolumn{2}{|c|}{ مشكل شديد } & \multicolumn{2}{|c|}{ 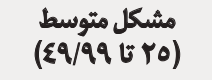 } & \multicolumn{2}{|c|}{ مشكل خفيف } & \multicolumn{2}{|c|}{ بدون مشكل } & \multirow{2}{*}{ راتندكان } \\
\hline درصد & تعداد & درصد & تعداد & درصد & تعداد & درصد & تعداد & درصد & تعداد & \\
\hline- & - & - & - & $q / \pi$ & if & $\Delta \Lambda$ & AV & $\pi / \& g$ & $p q$ & التوبوس \\
\hline- & - & $r$ & $r$ & $V / \pi T$ & 11 & s. & q. & $r .198$ & pe & ثاكسى \\
\hline- & - & 1 & $r$ & NTr & ta & $\Delta q$ & in & TI/Gg & 90 & مجموع \\
\hline
\end{tabular}

توانبخننى

جدول ب. فراواني و درصد ناتواني اسكلتي عضلاني نواحي شانه، بازو و دست در رانيدكان اتوبوس و ناكسي (مشكل فرد در انجام كارهاي روزمره)

\begin{tabular}{|c|c|c|c|c|c|c|c|c|c|c|}
\hline \multicolumn{2}{|c|}{ (1) ناتوان } & \multicolumn{2}{|c|}{ 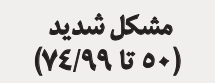 } & \multicolumn{2}{|c|}{ 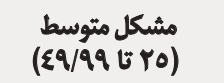 } & \multicolumn{2}{|c|}{ مشكل خُفيف } & \multicolumn{2}{|c|}{ بدون مشكل } & \multirow{2}{*}{ رانثدكان } \\
\hline هرصد & تعداد & ورصد & تعداد & ورصد & تعداد & ورصد & تعداد & درصد & تعداد & \\
\hline- & - & - & - & pleg & $v$ & FNES & $n$ & pelse & vo & اتوبوس \\
\hline- & - & .198 & 1 & $8 / 98$ & 1. & $r \Delta / T r$ & FA & $\mathrm{PV} / \mathrm{TH}^{\mathrm{T}}$ & $n$ & تاكسى \\
\hline- & - & $\cdot \pi$ & 1 & $\Delta / 98$ & IV & iv & ifi & iv & $|F|$ & مجموع \\
\hline
\end{tabular}

توانبخننىى 


\begin{tabular}{|c|c|c|c|c|c|c|c|c|c|c|}
\hline \multicolumn{2}{|c|}{ ناتوان } & \multicolumn{2}{|c|}{ 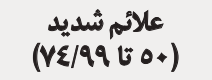 } & \multicolumn{2}{|c|}{ 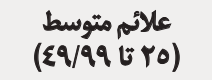 } & \multicolumn{2}{|c|}{ 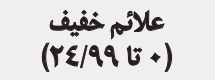 } & \multicolumn{2}{|c|}{ بدون علالثم } & \multirow{2}{*}{ راتندكًان } \\
\hline قرصد & تعداد & ورصد & تعداد & ورصد & تعداد & ورصد & تعداد & ورصد & تعداد & \\
\hline- & - & - & - & $r / M T$ & $\Delta$ & ef & $\vartheta$ & Mrles & 19 & اتوبوس \\
\hline- & - & - & - & F/\&8 & $\gamma$ & 8.188 & 91 & Me/gg & $\Delta r$ & تاكسى \\
\hline- & - & - & - & $f$ & ir & $\mathscr{E} / \pi$ & IAY & rife & 1.1 & مجموع \\
\hline
\end{tabular}

توانبخننى

\begin{tabular}{|c|c|c|c|c|c|c|c|c|c|c|}
\hline \multicolumn{2}{|c|}{ ناتوان } & \multicolumn{2}{|c|}{ 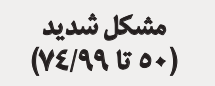 } & \multicolumn{2}{|c|}{ 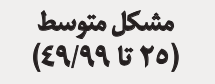 } & \multicolumn{2}{|c|}{ (م ماكل خفو } & \multicolumn{2}{|c|}{ بدون مشكل } & \multirow{2}{*}{ راتثدكان } \\
\hline درصد & تعداد & درصد & تعداد & درصد & تعداد & درصد & تعداد & درصد & تعداد & \\
\hline- & - & $\Delta / \pi T$ & $\wedge$ & IA & $r V$ & P.198 & 91 & re & $d f$ & التوبوس \\
\hline .198 & 1 & f & 8 & IA & $r V$ & 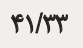 & er & $r e$ & $\Delta f$ & تاكسى \\
\hline.$/ \pi$ & 1 & rifs & if & M & of & il & "זr & rq & 1.1 & هجموع \\
\hline
\end{tabular}

توانبخننى

eleq Pleq

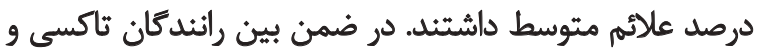

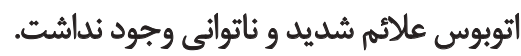

با توجه به جدول شماره ه، عس درصد از راندكان اتوبوس

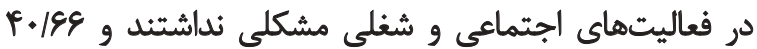

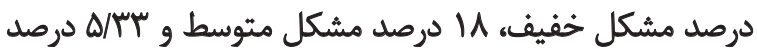

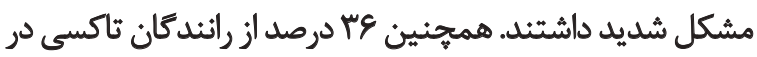

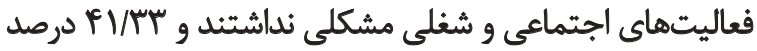

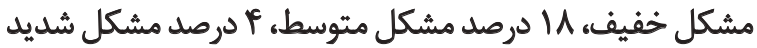

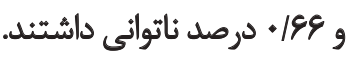

با توجه بيك جلول شماره ع، مقايسه ناتواني، علائم و عملكرد بين

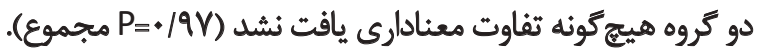

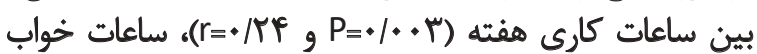

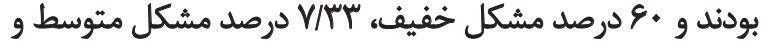

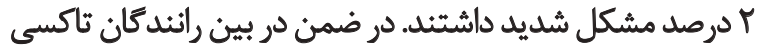
و اتوبوس ناتواني وجود نداشيت. شردي داشين.

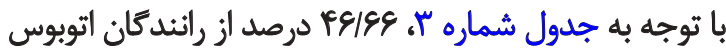

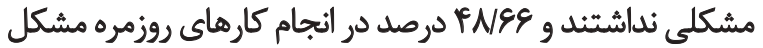

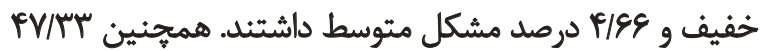

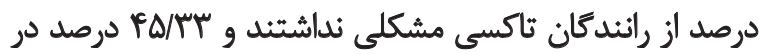

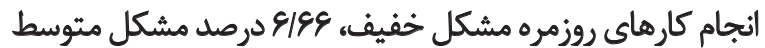

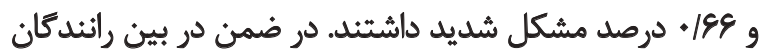
تاكسى و اتوبوس ناتوانى وجود نداشت.

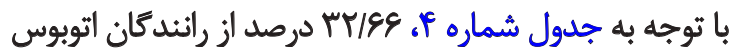

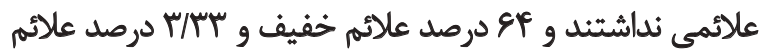

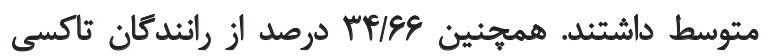

جدول \&. مثايسه ناتوائى، علائم و عملكرد بين دو گروه رائندكان اتوبوس و تاكسى

\begin{tabular}{|c|c|c|c|c|}
\hline مجموع & عملكرد & عالأم & 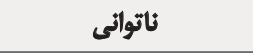 & \\
\hline مياتكين × انحراف معيار & ميانكين د أحراف معيار & ميانكين × انحراف معيار & ميانكين × انحراف معيار & \\
\hline$N M Y \pm 1 \cdot / r$ & $|r / 9| \pm \mid \& / 9 r$ & $\Delta / \cdot Y \pm V / q Y$ & $\Delta / \cdot Y \pm V / a Y$ & اتوبوس \\
\hline$N T V \pm 11 / T E$ & IT/RTIIF/RT & $\mathbb{H} / \pi \pm \Delta / \pi$ & $\Delta / U \pm 1 . / 1 V$ & 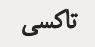 \\
\hline .194 & $\cdot / \Lambda_{0}$ &.$/ T 8$ &.$/ 1$. & $P$ \\
\hline
\end{tabular}

توانبخننى 


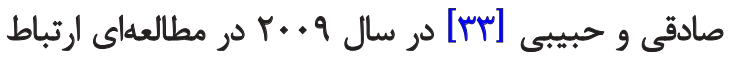

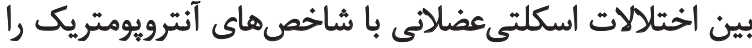

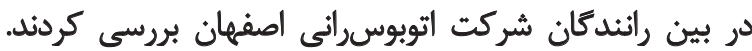

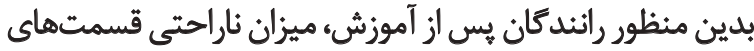

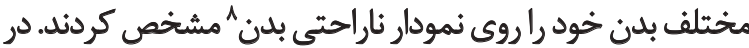

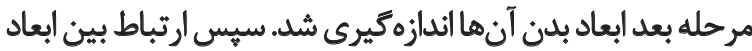

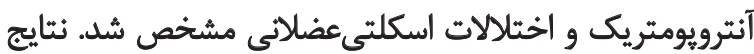

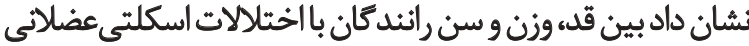

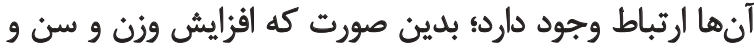

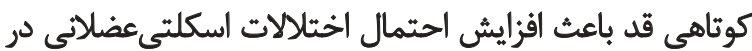
راننده خواهد شد.

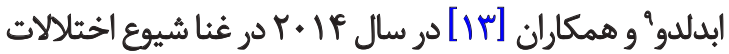

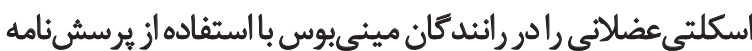

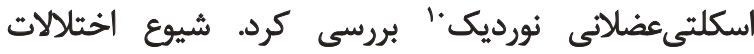

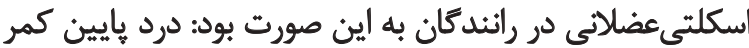

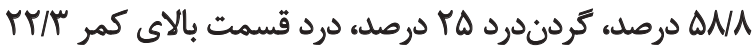

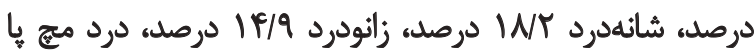

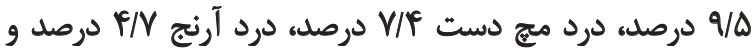

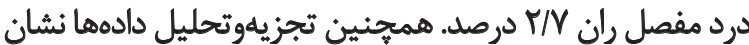

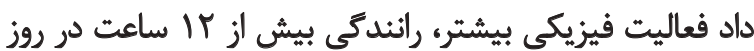

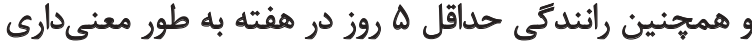
با شيوع اختلالات اسكلتى عضلانى در بين رانئهها ارتباط هاط دارد. نتايج مشاهدهده در مطالعات ذكرشده با نتايج بلدست آمده

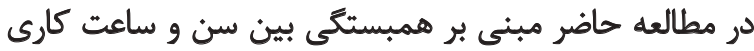

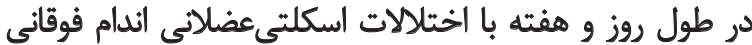
رانيدكان اتوبوس همخوانى دارد.

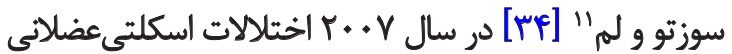

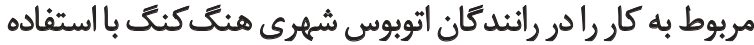

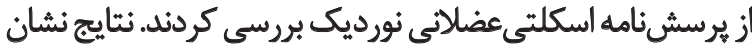

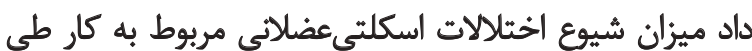

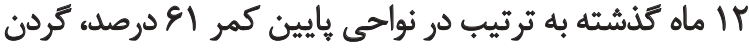

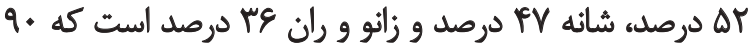

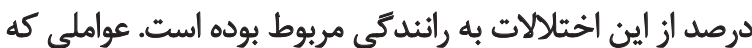

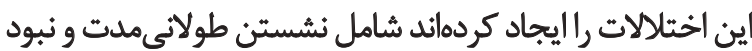

$$
\text { هماهنتى آنترويومتريك بوده است. }
$$

همانطور كه در اين مطالعه نيز مشاهده شد، شانه يكى از

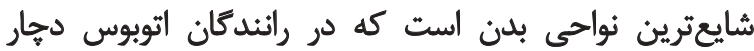

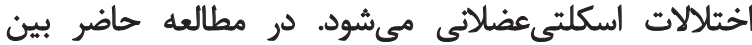
ساعت كارى در هفته با اختلالات اسكلتىعضلانى انداني اندام فوقانى

8. Body Discomfort Chart (BDC)

9. Abledu

10. Nordic Musculoskeletal Questionnaire (NMQ)

11. Szeto and Lam

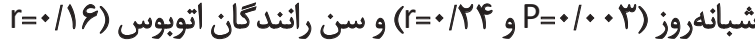

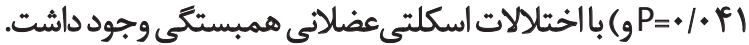
همجنين بين ساعات خواب شبائهروز و اختلالات اسكلتى عضلانى هني

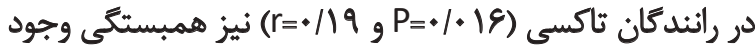

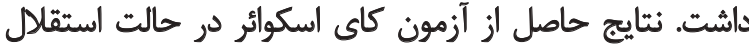
كروها نشان داد بين ميانكين سطح تحصيلات (P= (P) (P)،

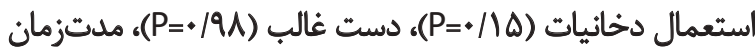
ورزش كردن (P=ء) و شيوع اختلالات اسكلتي عضلاني ارتباط

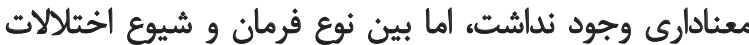

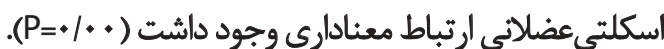

ث)

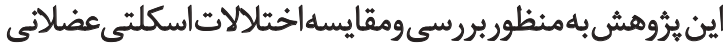
نواحى شائه، بازو و دست در دو كروه رائندكان تاكسى واتوبوس

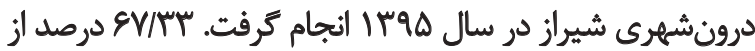

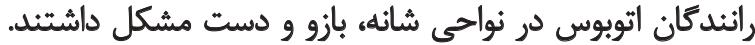

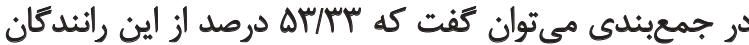
در انجام كارهاى روزمره مقادير متفاوتى ناتوانى داشتئند

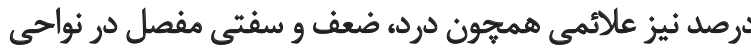

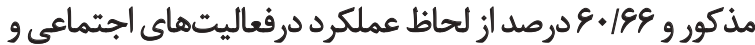

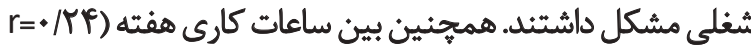

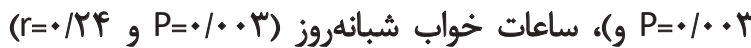

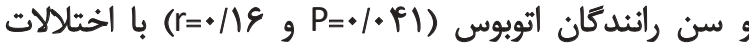

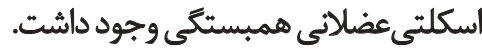

از آنجا كه مطالعات صورت كرفته روى رانندكان، روش اجراو

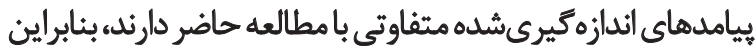

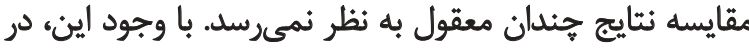

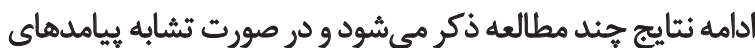

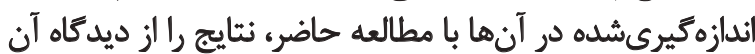
يامدها مقايسه مي كنيم.

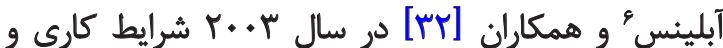

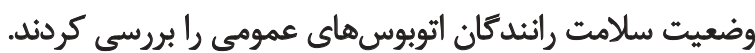

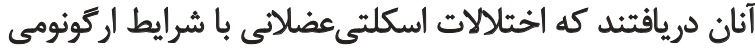
نامناسب در محل كار، ساعات كار طولاتئ در روز، سن و سابقه باتل كارى مرتبط است.

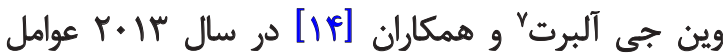

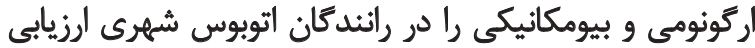

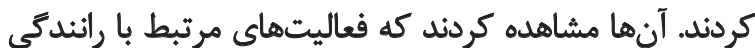

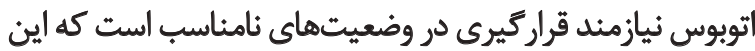
وضعيت در ساعات بايانى كار تشديد مي دئودي.

6. Obelinis

7. Wayen J. Albert 


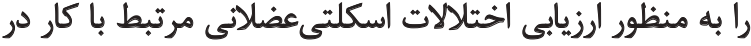

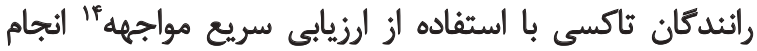

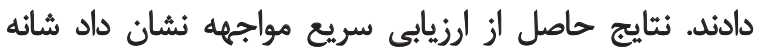

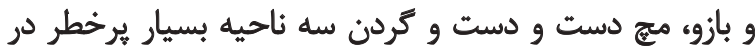

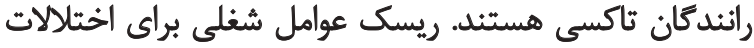

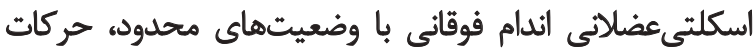

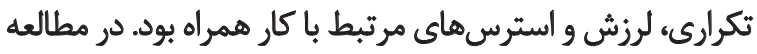

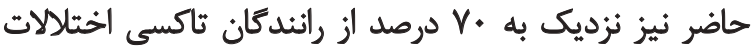

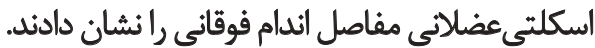

بررسى حاضر نشان داد از لحاظ ميزان ناتواني، شدت علاتئم

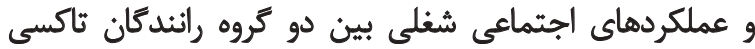

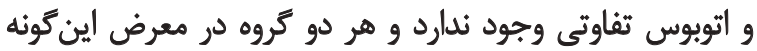

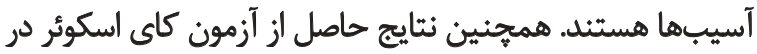

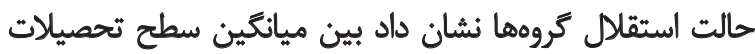

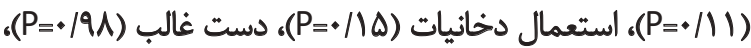

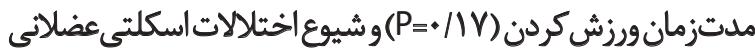

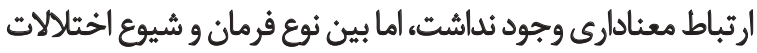

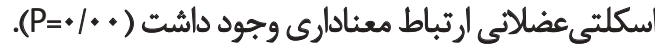

\section{نتيجلئيرى}

بهلوركلى اين تحقيق نشان داد هر دو كروه رانيدكان از

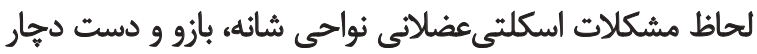

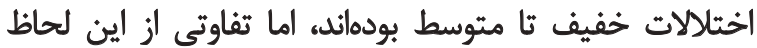

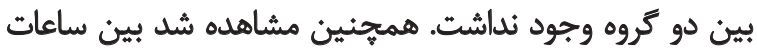

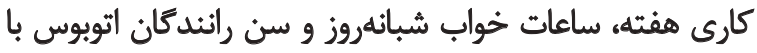

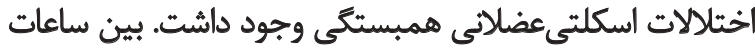

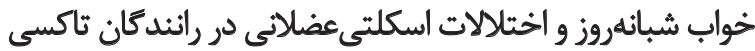

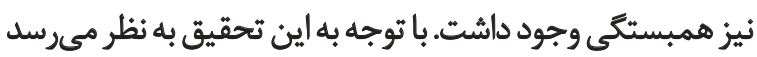

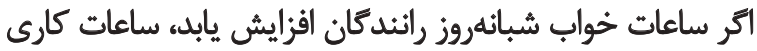

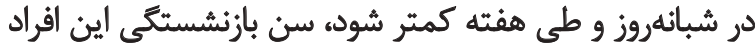

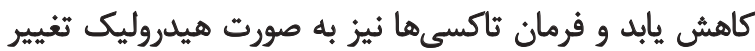

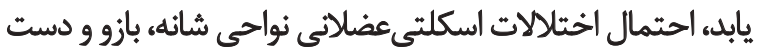

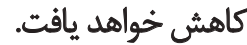

يبشنهاد مىشود در تحقيقهاى آتى، بررسى جامعترى روى دئ

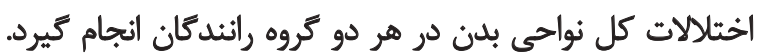

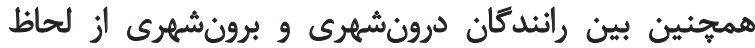

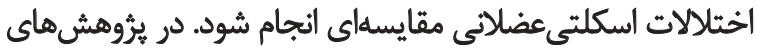

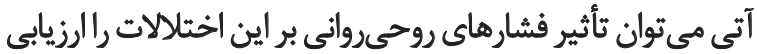

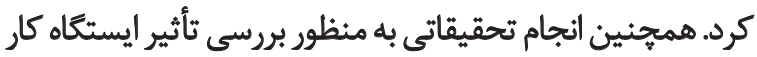

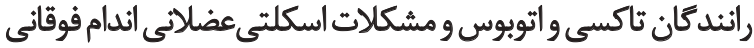

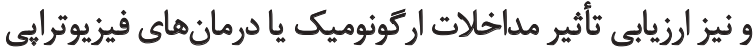

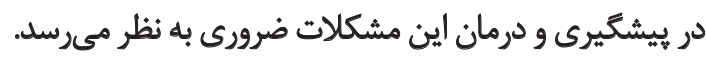

14. Quick Exposure Check (QEC)
در راندكًان اتوبوس همبستكى وجود داشت. به نظر مىرسد

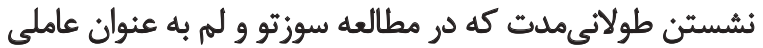

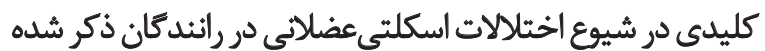

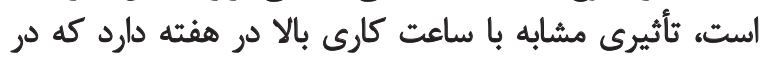

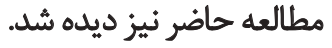

به طور كلى بr/49 درصد از رانندكان ثاكسى در نواحى شانه.

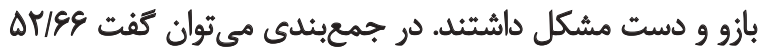

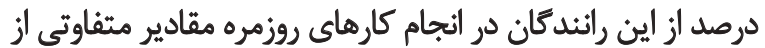

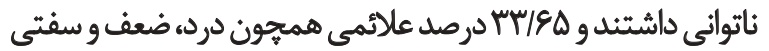

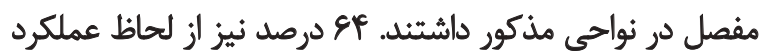

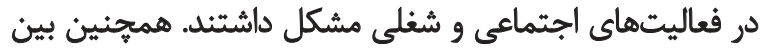

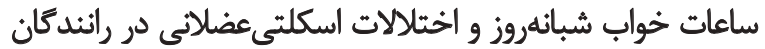

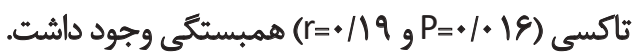

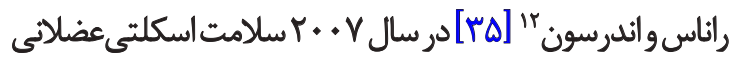

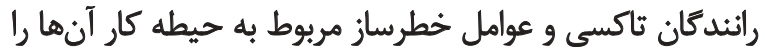

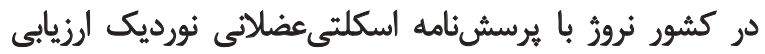

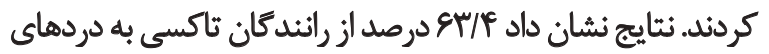

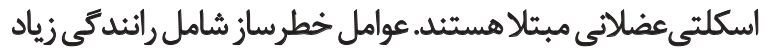

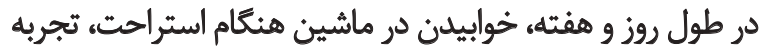

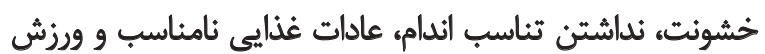

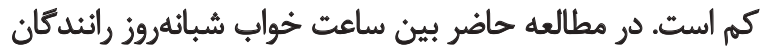

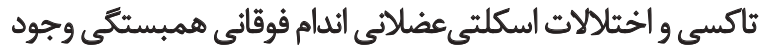

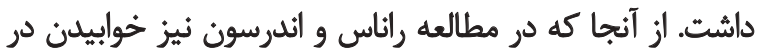

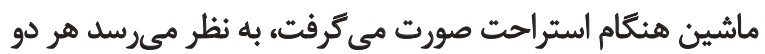

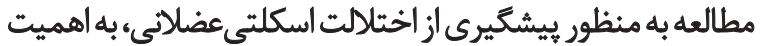
ميزان كافى و كيفيت مطلوب خواب در رانندكان اشاره دارندانداني

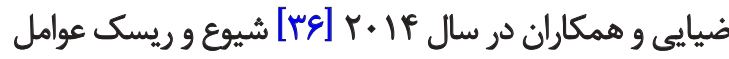

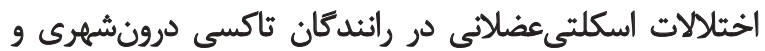

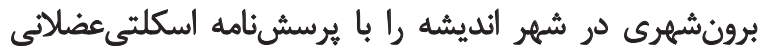

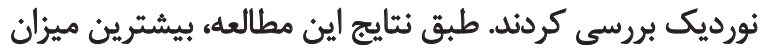

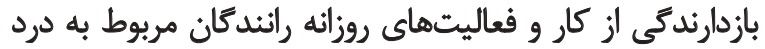

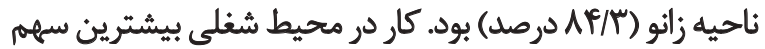

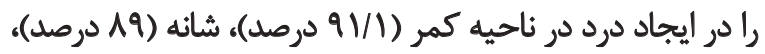

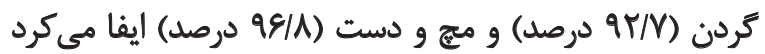

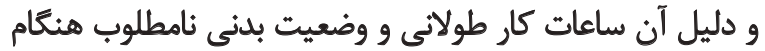

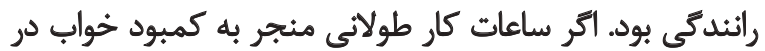

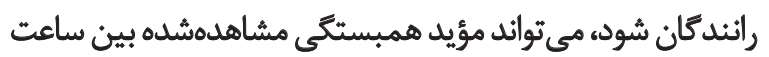

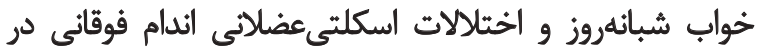
رانيدكان تاكسى در مطالعه حاضر باشد.

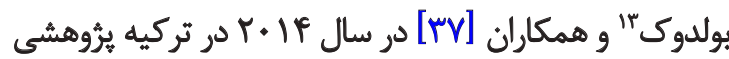




$$
\text { تشكر و قدرداني }
$$

مقاله حامى مالى ندارد. 


\section{References}

[1] Khandan M, Ataeimanesh L, Eyni Z, Khosravi Z, Biglari H, Koohpaei A, et al. Relationship between job content and demographic variables with musculoskeletal disorders among nurses in a university hospital, Qom Province, 2014. Research Journal of Applied Sciences. 2016; 11(7):547-53. doi: 10.3923/ rjasci.2016.547.553

[2] Nunes IL. FAST ERGO_X-a tool for ergonomic auditing and work-related musculoskeletal disorders prevention. Work. 2009; 34(2):133-48. doi: 10.3233/WOR-2009-0912

[3] Yektaee T, Tabatabaee Ghomshe F, Piri L. [The effect of ergonomic principles education on musculoskeletal disorders among computer users (Persian)]. Archives of Rehabilitation. 2013; 13(4):108-16

[4] Fredriksson K, Bildt C, Hägg G, Kilbom Å. The impact on musculoskeletal disorders of changing physical and psychosocial work environment conditions in the automobile industry. International Journal of Industrial Ergonomics. 2001; 28(1):31-45. doi: 10.1016/s0169-8141(01)00011-7

[5] Matiin M, Ebrahimi II, Shater Zadeh MJ, Salavati M, KazemNezhad A. [Muscale activation pattern of shoulder girdle during some routine physical therapy exercises of upper limb (Persian)]. Archives of Rehabilitation. 2010; 11(2):49-58.

[6] Lei L, Dempsey PG, Xu J, Ge L, Liang Y. Risk factors for the prevalence of musculoskeletal disorders among chinese foundry workers. International Journal of Industrial Ergonomics. 2005; 35(3):197-204. doi: 10.1016/j.ergon.2004.08.007

[7] K Abledu J. Multiple logistic regression analysis of predictors of musculoskeletal disorder and disability among bank workers in Kumasi. Journal of Ergonomics. 2012; 2(4):111-5. doi: $10.4172 / 2165-7556.1000111$

[8] Azari GR, Davuian Talab AH. [Comparison of burnout and musculoskeletal disorders among computer users and office workers (Persian)]. Archives of Rehabilitation. 2012; 12:38-46.

[9] Khandan M, Eyni Z, Manesh LA, Khosravi Z, Biglari H, Koohpaei A. Relationship between musculoskeletal disorders and job performance among nurses and nursing aides in main educational hospital in qom province, 2014. Research Journal of Medical Sciences. 2016; 10(4):307-12.

[10] Omidianidost A, Hosseini S, Jabari M, Poursadeghiyan M, Dabirian M, Charganeh S, Yarmohammadi $H$. The relationship between individual, occupational factors and LBP (Low Back Pain) in one of the auto parts manufacturing workshops of Tehran in 2015. Journal of Engineering and Applied Sciences. 2016; 11(5):1074-7.

[11] Poursadeghiyan M, Tabar A. Comparing the posture assessments based on RULA and QEC methods in a carpentry workshop. Research Journal of Medical Sciences. 2016; 10(3):80-3.

[12] Abledu JK, Offei EB, Abledu GK. Predictors of work-related musculoskeletal disorders among commercial minibus drivers in Accra Metropolis, Ghana. Advances in Epidemiology. 2014; 2014:1-5. doi: 10.1155/2014/384279

[13] Abledu JK, Offei EB, Abledu GK. Occupational and personal determinants of musculoskeletal disorders among urban taxi drivers in Ghana. International Scholarly Research Notices. 2014; 2014:1-5. doi: 10.1155/2014/517259

[14] Albert WJ, Everson D, Rae M, Callaghan JP, Croll J, Kuruganti U. Biomechanical and ergonomic assessment of urban transit operators. Work. 2014; 47(1):33-44. doi: 10.3233/WOR-131683

[15] Alperovitch Najenson D, Santo Y, Masharawi Y, Katz Leurer M, Ushvaev D, Kalichman L. Low back pain among professional bus drivers: ergonomic and occupational-psychosocial risk factors. Israel Medical Association Journal. 2010; 12(1):26-31. PMID: 20450125

[16] Bovenzi M, Rui F, Negro C, D’Agostin F, Angotzi G, Bianchi S, et al. An epidemiological study of low back pain in professional drivers. Journal of Sound and Vibration. 2006; 298(3):514-39. doi: 10.1016/j.jsv.2006.06.001

[17] Magnusson ML, Pope MH, Wilder DG, Areskoug B. Are occupational drivers at an increased risk for developing musculoskeletal disorders. Spine. 1996; 21(6):710-7. doi: 10.1097/00007632199603150-00010

[18] Robb MJM, Mansfield NJ. Self-reported musculoskeletal problems amongst professional truck drivers. Ergonomics. 2007; 50(6):814-27. doi: 10.1080/00140130701220341

[19] Madhan Chandran SY. Are bus drivers at an increased risk for developing musculoskeletal disorders? An Ergonomic Risk Assessment Study. Journal of Ergonomics. 2015; s3. doi: 10.4172/2165-7556.s3-011

[20] Mazloumi A, Mohammadreze F. Ergonomic evaluation of interior design of Shoka vehicle and proposing recommendations for improvement. Work. 2012; 41(Supplement 1):1477-85. doi: 10.3233/WOR-2012-0341-1477

[21] Anderson R. The back pain of bus drivers. Spine. 1992; 17(12):1481-8. doi: 10.1097/00007632-199212000-00007

[22] Backman AL, Jarvinen E. Turnover of professional drivers. Scandinavian Journal of Work, Environment \& Health. 1983; 9(1):36-41. doi: $10.5271 /$ sjweh. 2445

[23] Feickert D, Forrester K. Stress factors in urban public transport work. Paper presented at Conference on the Working Environment in Urban Public Transport. 12 January 1983, Stockholm, Sweden.

[24] Kompier MAJ. Work and health of city bus drivers. Delf: Eburon; 1988.

[25] Kompier MA, Di Martino V. Review of bus drivers' occupational stress and stress prevention. Stress and Health. 1995; 11(1):253 62. doi: 10.1002/smi.2460110141

[26] Meifort J, Reiners H, Schuh J. [Working conditions of bus and tram drivers of Dortmunder Stadtwerke Aktiengesellschaft (series of publications on occupational safety) (German)]. Berlin: Bundesanstalt für Arbeitsschutz und Unfallforschung; 1983.

[27] Chen JC, Chang WR, Chang W, Christiani D. Occupational factors associated with low back pain in urban taxi drivers. Occupational Medicine. 2005; 55(7):535-40. doi: 10.1093/occmed/ kqi125

[28] Yazdani F, Dehkordi F. Occupational therapy intervention in combination flexor tendon and peripheral nerve injury $10 \mathrm{pa}-$ 
tients with disabilities of arm, shoulder and hand. Iranian Rehabilitation Journal. 2013; 11:21-6.

[29] Abdollahi I, Mohseni Bandpei M, Firoozi S. [The prevalence of upper extremity disorders in physiotherapists of Tehran City (Persian)]. Archives of Rehabilitation. 2012; 12(4):47-53.

[30] Khandan M, Maghsoudipour M, Vosoughi S. Ergonomic behavioral and workers' safety perception in Khuzestan Petrochemical Company. Iranian Rehabilitation Journal. 2012; 10:26-30.

[31] Mousavi SJ, Parnianpour M, Abedi M, Askary Ashtiani A, Karimi A, Khorsandi A, et al. Cultural adaptation and validation of the Persian version of the Disabilities of the Arm, Shoulder and Hand (DASH) outcome measure. Clinical Rehabilitation . 2008; 22(8):749-57. doi: 10.1177/0269215508085821

[32] Obelenis V, Gedgaudienè D, Vasilavičius P. Working conditions and health of the employees of public bus and trolleybus transport in Lithuania. Medicina. 2003; 39(11):1103-9.

[33] Sadeghi N, Habibi E. The survey of relation between Musculoskeletal Disorders and Anthropometric Indices in the bus drivers in Isfahan. Iran Occupational Health. 2009; 6(1):6-14.

[34] Szeto GPY, Lam P. Work-related musculoskeletal disorders in urban bus drivers of Hong Kong. Journal of Occupational Rehabilitation. 2007; 17(2):181-98. doi: 10.1007/s10926-007-9070-7

[35] Raanaas RK, Anderson D. A questionnaire survey of Norwegian taxi drivers' musculoskeletal health, and work-related risk factors. International Journal of Industrial Ergonomics. 2008; 38(3-4):280-90. doi: 10.1016/j.ergon.2007.10.017

[36] Ziaei M, Izadpanah S, Sharafi K, Barzegar Shangol A. Prevalence and risk factors of musculoskeletal disorders in inside and outside-city taxi drivers Andisheh city, 2011. Razi Journal of Medical Sciences. 2014; 21(118):41-50.

[37] Bulduk EÖ, Bulduk S, Süren T, Ovalı F. Assessing exposure to risk factors for work-related musculoskeletal disorders using Quick Exposure Check (QEC) in taxi drivers. International Journal of Industrial Ergonomics. 2014; 44(6):817-20. doi: 10.1016/j. ergon.2014.10.002 
